\title{
Cirrhosis: Morphologic Dynamics for the NonMorphologist
}

\author{
Henry D. Appelman, MD \\ Cirrhosis is an irreversible end-stage liver disease characterized by septate \\ scars dividing a distorted liver into nodules. It generates through a se- \\ quence of dynamic changes and once it develops, it may alter its ap- \\ pearance through variations in secondary factors, such as injury and \\ nutrition. The different classification schemes have, unfortunately, only \\ served to make cirrhosis static in our thinking. Stationary morphologic \\ characteristics are of value only if they can be correlated with etiology.
}

In the simplest sense, cirrhosis is a diffuse, irreversible late-stage chronic liver disease characterized morphologically by septate scars separating nodules of liver parenchyma, severely distorting its architecture (Figure 1), and clinically; by aberrations involving hepatocellular function and the hepatic vascular system. The most complex aspect of cirrhosis is its definition. It is both a clinical disease, appearing as a sequence of changing physical phenomena, and a histologic disease recognized as a static entity viewed at a given point in time. Yet these two views do not always coincide.

This lack of agrecment, between clinicians and pathologists about the meaning of cirrhosis is the result of several factors. First; morphologists tend to hold to rigid criteria for their diagnosis of cirrhosis. Generally, they demand that there be both septate scars, criss-crossing the hepatic parenchyma, and regenerative nodules of residual liver tissue. Both of these will be defined in more detail shortly. However, there are chronic hepatic diseases which may present with clinical features of cirrhosis and yet, when samples of these livers are viewed microscopically, the previously mentioned rigid criteria of histologic cirrhosis may not be fulfilled. Thus, there may be clinical cirrhosis without morphologic cirrhosis (Figure 2).

From the Department of Pathology, University of Michigan Medical School, Ann Arbor, Mich.
Second, there are the few cases of cirrhosis discovered incidentally at autopsy which were not clinically apparent. For some unexplained reason, these patients were able to compensate to a degree sufficient to mask their disease clinically--essentially they had subclinical cirrhosis roughly comparable to the very common subclinical viral hepatitis. In these cases, there was morphologic cirrhosis without clinical cirrhosis

Nevertheless, in spite of these exceptions, in most cases of cirrhosis the clinical features of the disease correlate well with morphologic findings.

We can view cirrhosis as an advanced diffuse chronic inflammatory disease. Chronicity refers to both the time over which the disease develops, generally measured in years, and to the type of inflammatory reaction seen microscopically-a reaction characterized by variable degrees of scarring, cellular infiltration and attempts at regeneration by the liver tissue. This chronic inflammation is assumed to be a response to liver injury which resulted in tissue death or necrosis. The injurious stimuli include a variety of infectious agents such as: viruses of hepatitis; toxic substances, one of which may be alcohol; drugs or poisons, such as carbon tetrachloride; a group of factors which might be termed mechanical, in part, including circulatory and biliary obstructive; several metabolic 


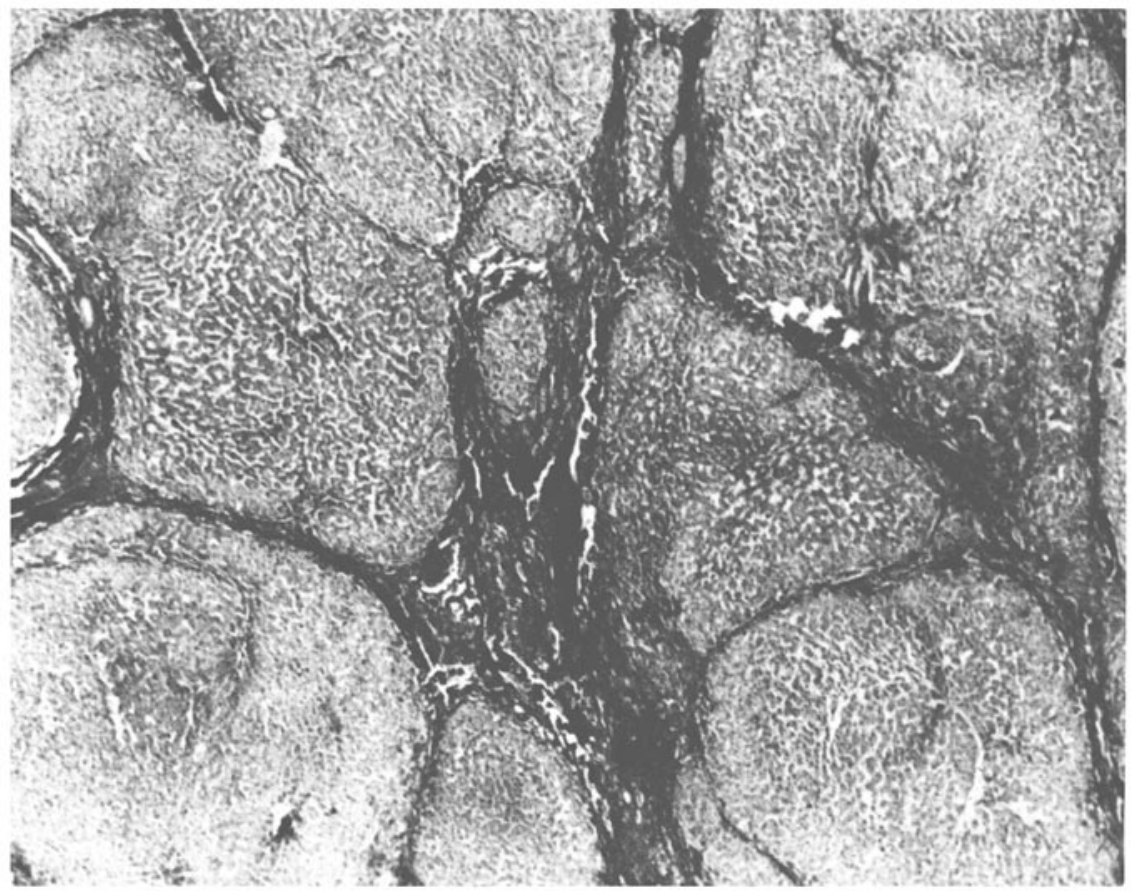

Fig 1. Cirrhosis, characterized by fibrous septa subdividing the hepatic parenchyma into hyperplastic subunits or regenerative nodules (Masson trichrome $\times 30$ ).

aberrations or "inborn errors," such as galactosemia and Wilson's disease; and, finally, the great unknown-the cryptogenic factors which, in our present state of knowledge, unfortunately must remain the explanation for many cases of cirrhosis. Perhaps some of these factors act in combination to stimulate the development of cirrhosis.

The degree or extent of insult or injury necessary for cirrhosis to begin is not understood. Viral hepatitis, a common disease which severely and diffusely disturbs the hepatic parenchyma only infrequently has been proven a cause of cirrhosis (1). On the other hand, many cases of cirrhosis occur in patients who have no clinical history of previous acute liver injuries or exposure to hepatotoxins.

Our assumption, however, is that injury or injuries result in hepatocellular necrosis, the basic lesion. From this point, a multifaceted reaction begins which involves the inflamma- tory process, the extent of necrosis, and the liver's attempt to replace itself by regeneration. We recognize these changes microscopically, but a clear-cut understanding of the mechanisms which cause them is lacking. Therefore, most of our discussion of the genesis of cirrhosis is based upon considerable speculation. $\Lambda$ postulated scheme for the dynamics of its development is presented in Figure 3. Since these concepts are changing rapidly, one should

\section{Cirrhosis}

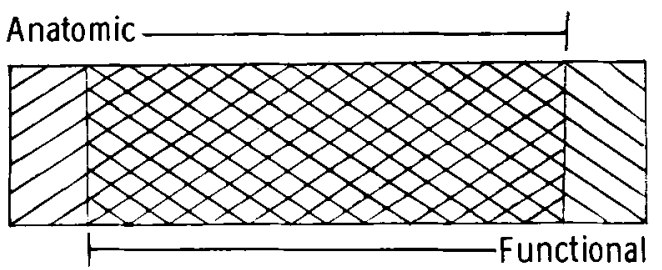

Fig 2. Criteria for diagnosis of cirrhosis. 


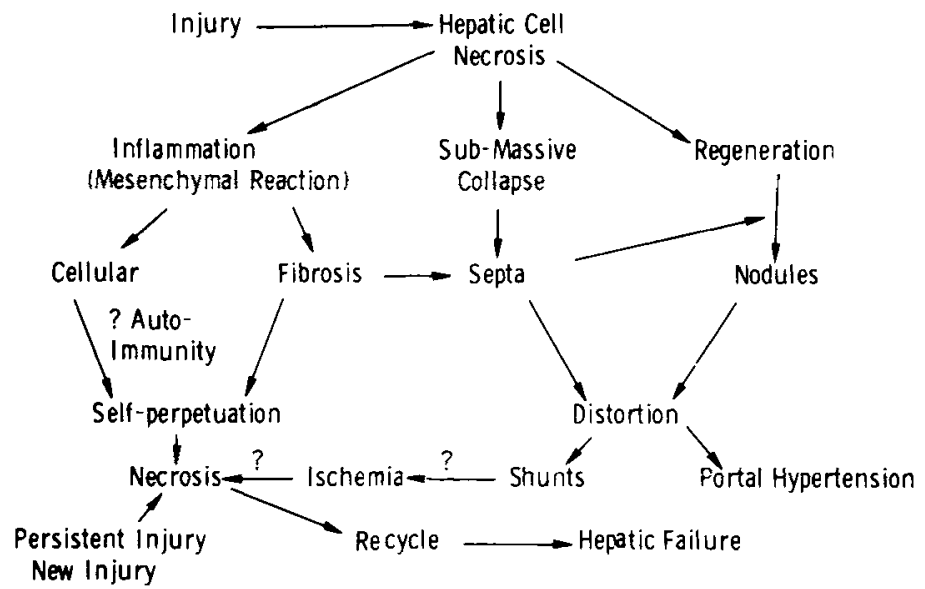

Fig 3. The development of cirrhosis.

expect alterations in this scheme, much of which is a simplistic compendium of the complex observations of Rubin and Popper (2). There are three aspects of this scheme which we may consider separately, but which are actually quite interrelated.

\section{The Inflammatory-Fibrosis-Perpetuation Cycle (Figure 4)}

Hepatocellular necrosis is assumed to lead to a mesenchymal reaction or inflammation which may be exudative or cellular with lymphocytes, histiocytes and plasma cells (Figure 5,) or productive with fibrosis (Figure 6). In the development of cirrhosis, both seem to occur. We can speculate that these inflammatory cells are part of the body's response, perhaps immunologic, to necrotic tissue or to toxic metabolic products from injured hepatocytes. In many respects, the cellular reaction resembles that seen in delayed hypersensitivity as in transplant rejection. We do not know if the presence of these cells creates further necrosis or if they simply respond to preexisting necrosis and cause little additional injury. However, we suspect their presence is

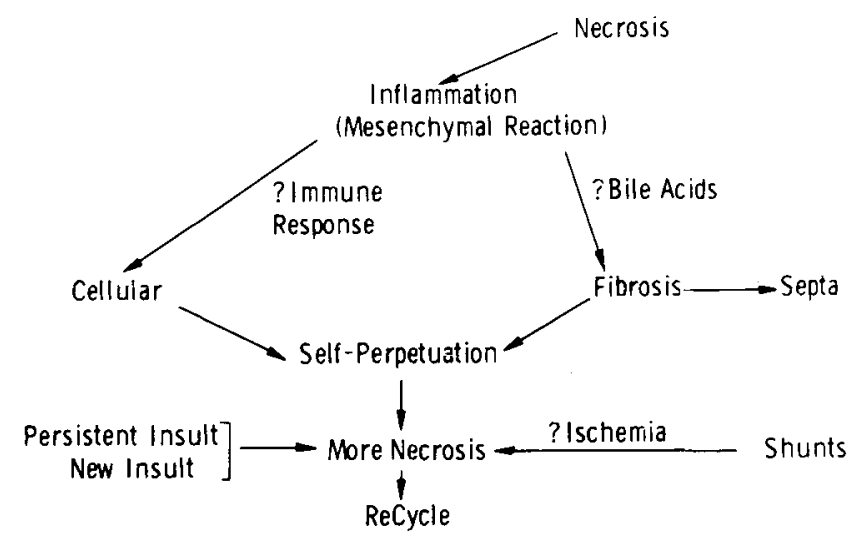

Fig 4. The cycle of inflammation-fibrosis-perpetuation. 


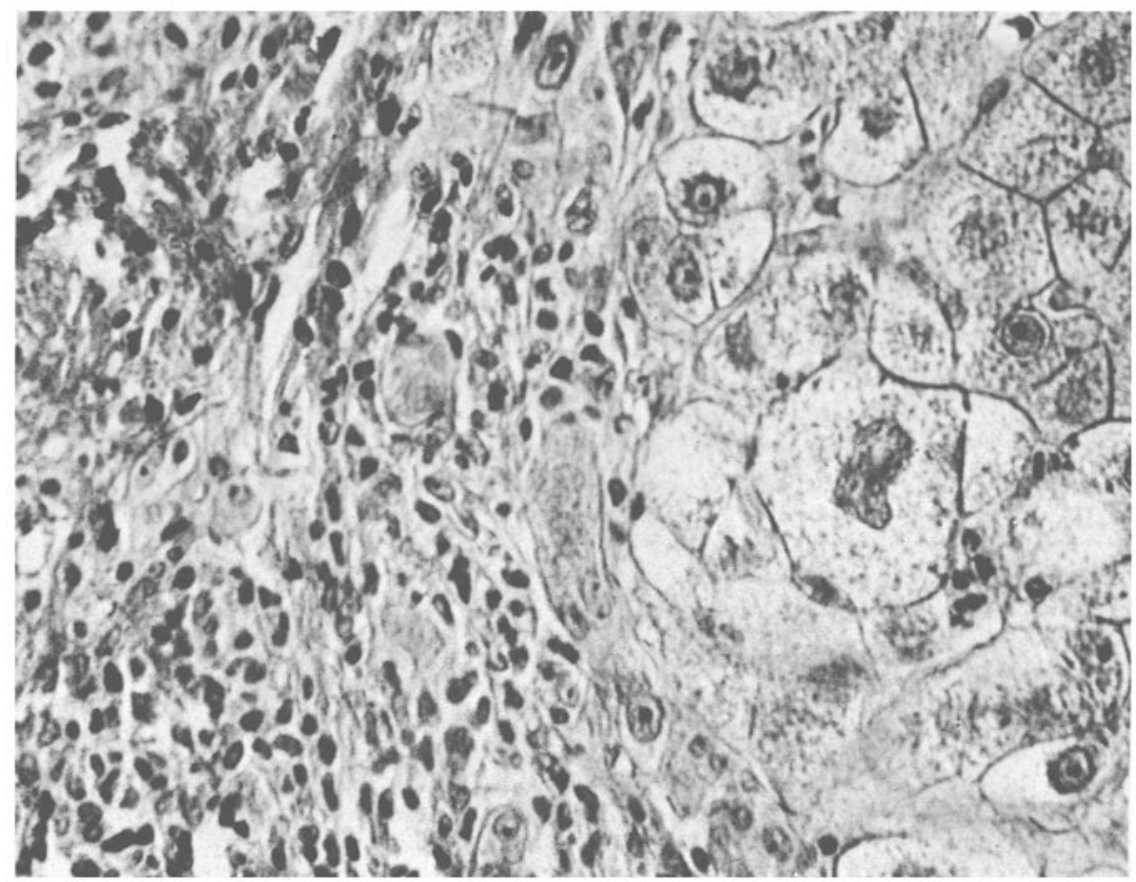

Fig 5. The cellular or exudative inflammatory component: at the right is the edge of a nodule, and at the left is an infiltrate of lymphocytes, histocytes and plasma cells which partly surround hepatocytes in the center of the field (Masson trichome, $\times 281$ ).

an index to activity of the disease, especially if they involve the peripheral plate of hepatic cells adjacent to the portal tracts (3). It is believed that fibrosis or collagenous proliferation is important in causing further damage (3). In fact, it may be the single most important determinant of perpetuation. In histologic sections of cirrhotic livers, one can often find hepatocytes trapped by collagen fibers (Figure 6).

The cause of fibrosis is also poorly understood (4). Popper suggested that retained bile acids may be the stimulis to fibrosis (3). There is a variety of patterns in which fibroblastic proliferation might appear. It might begin mainly in periportal areas (5), in centrilobular zones about the central veins (6) or in areas of parenchymal loss (collapse), the result of large regions of necrosis. As fibrosis proceeds, septal scars appear, subdividing the parenchyma into units of varying sizes. Such septa may link adjacent portal tracts (Figure 7), extend from portal tracts to centrilobular zones (Figure 8) or bridge adjacent central veins.

The significant consequence, then, of the total mesenchymal reaction is selfperpetuation of the process, keeping it active and directed inexorably toward the final stage, namely, cirrhosis (2). Other factors, however, might contribute to this perpetuation. The initial insult to the liver may persist or a new insult, such as a different toxic or second infectious stimulus, might supervene. The disturbed hepatic vascular pattern which is developing, might cause variable degrees of local ischemia in different parts of the liver and lead to additional necrosis. Whatever the mechanisms of perpetuation 


\section{CIRRHOSIS}

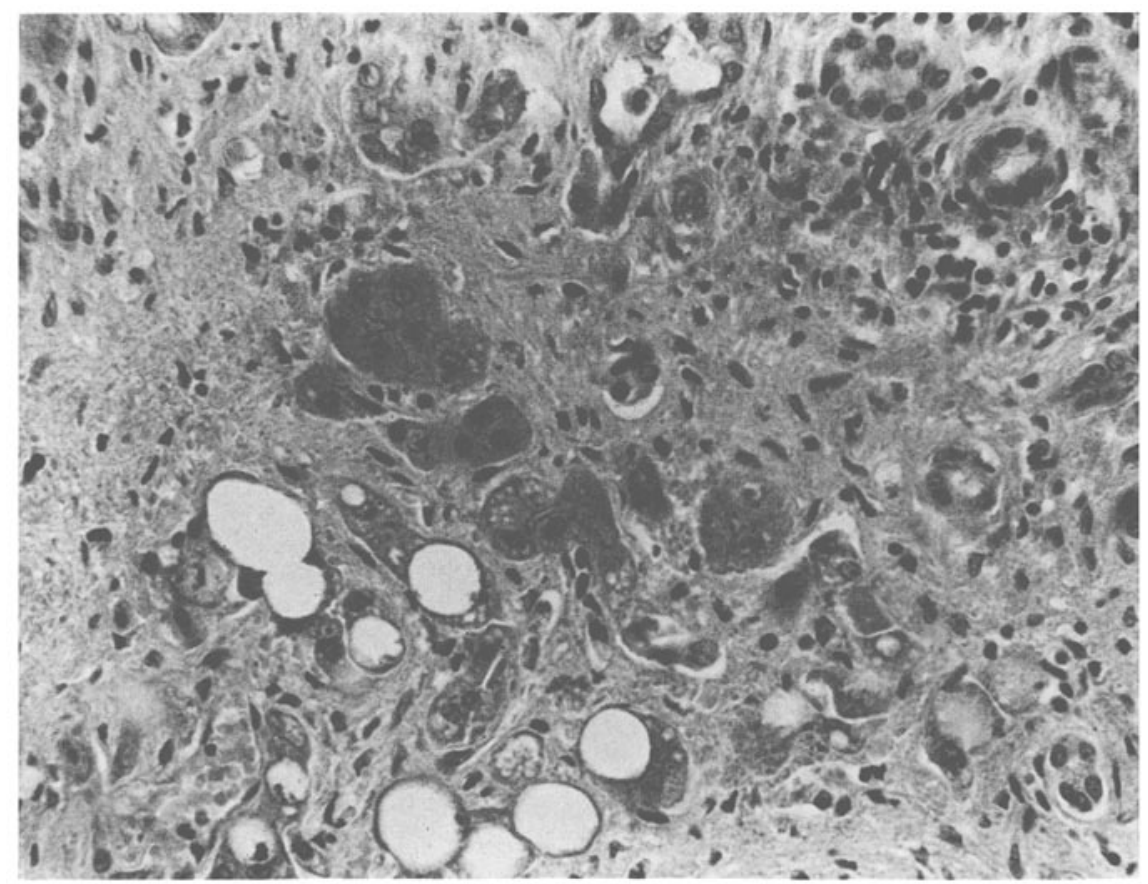

Fig 6. The productive or fibrosis aspect of cirrhosis: the fibrillar background in this field is collagen which surrounds small groups of hepatocytes and single hepatocytes, several of which contain large lipid vacuoles (Masson trichome, $\times 281$ ).

are, they produce additional necrosis, setting the stage for generations of cycles of the entire mesenchymal reaction.

\section{Collapse and Septa Formation (Figure 9)}

The term "collapse," a favorite among hepatopathologists, refers to the condensation of stromal fibers, reticulin and collagen, which previously supported parenchyma that has disappeared. This lost parenchyma apparently became necrotic and cellular debris was cleared, leaving the reticulin framework relatively intact but unsupported. Such areas of necrosis leading to collapse vary considerably in extent from small scattered foci, seen in most cases of acute viral hepatitis (Figure 10) to the tracts of subacute hepatic necrosis (7) and to fairly large areas of parenchyma (Figure 11). Such large foci are spoken of as "submassive necrosis," essentially, that degree of necrosis too great to regenerate parenchyma in the area involved, yet not great enough to kill the patient $(8,9)$. Such large zones of necrosis are very rarely encountered in biopsy material from a living patient. Certainly, we see large areas of necrosis in autopsy liver specimens from patients with fulminant hepatitis; however, in such cases, the collapse is not "submassive" but truly massive or total.

Nevertheless, assuming that such large necrotic lesions do occur, then, since regeneration is impossible at that time, fibers supporting necrotic liver cells must collapse forming septa of collapse. These septa seem to mature and eventually appear as elongated collagenized scars (8, Figure 7). Simultaneously, the fibroblastic component of the mesenchymal reaction, previously discussed, contributes to scarring with the formation of additional septa. As these septa form and begin to subdivide the liver, the architectural distortion of cirrhosis becomes visi- 


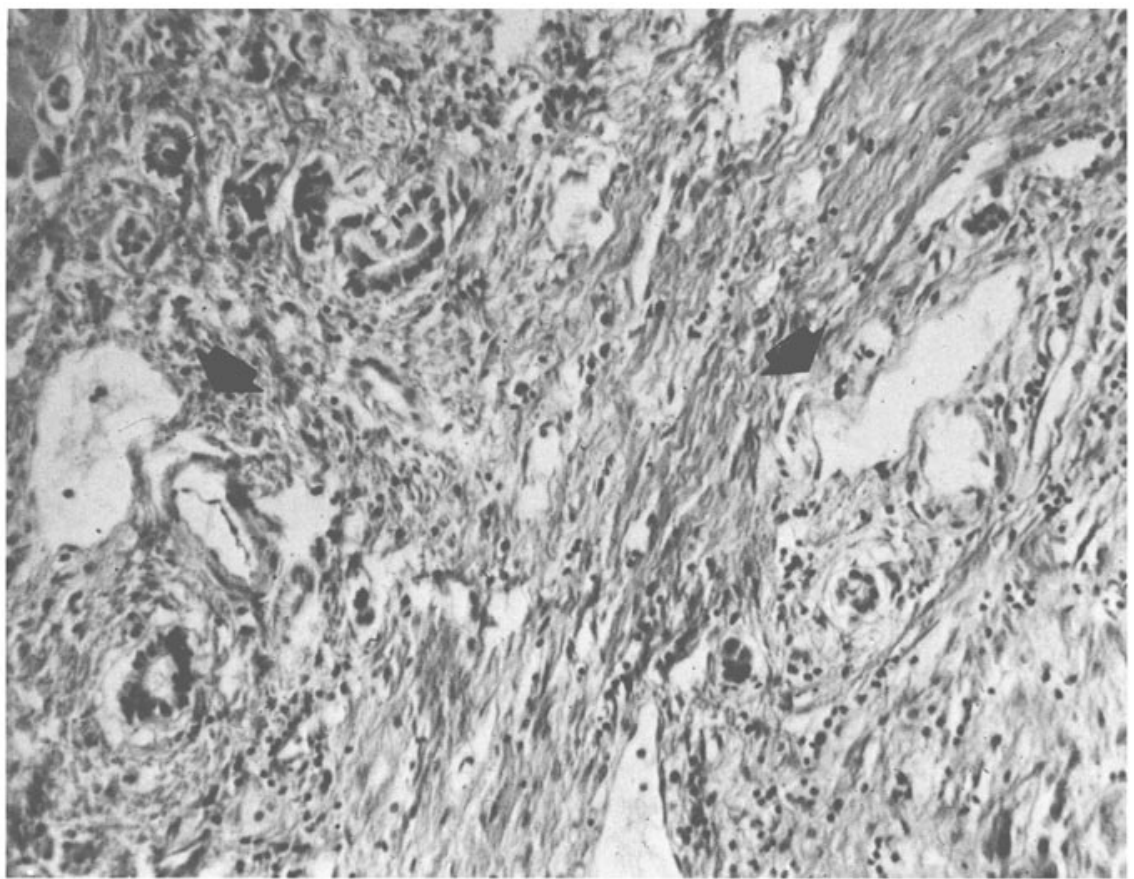

Fig 7. Two portal tracts (arrows) are connected by a collagenous scar where parenchyma formerly existed. This is the end stage of collapse in this area (Hematoxylic and lesia. $\times 180$ ).

ble. Within these scars, communications between afferent and efferent vascular systems develop, effectively bypassing parts of the parenchyma, thus adding an ischemic element to an already damaged liver (4).

\section{Regeneration and Nodule Formation (Figure 12)}

One suspects that an injured liver attempts to maintain its integrity. Therefore, necrosis of hepatocytes is followed by regeneration. In the cirrhotic livers, regeneration becomes excessive, resulting in nodules of proliferating hepatocytes (Figure 13). The reasons behind excessive regeneration or hyperplasia are not clear. Possibly, this is partly an attempt at anatomic compensation to improve or maintain the function of an imperfectly functioning liver.

\section{Architectural Chaos-The Cirrhotic Liver}

The three facets: a) inflammation with cellular and fibroblastic components; b) collapse and septation, and c) nodular regeneration, are simultaneously 'occuring processes which contribute to each other. They result in a liver divided into parenchymal masses of different sizes by scars of variable widths and directions (Figure 1). Patterns of scarring and nodularity possibly depend upon the type of insult that stimulated the process. Such patterns provide the basis for most classification schemes of cirrhosis.

The hepatic vascular system becomes severely distorted. Shunts between hepatic artery, and portal and hepatic vein branches decrease perfusion of remaining hepatic cells, thereby hindering hepatic function and probably creating 


\section{CIRRHOSIS}

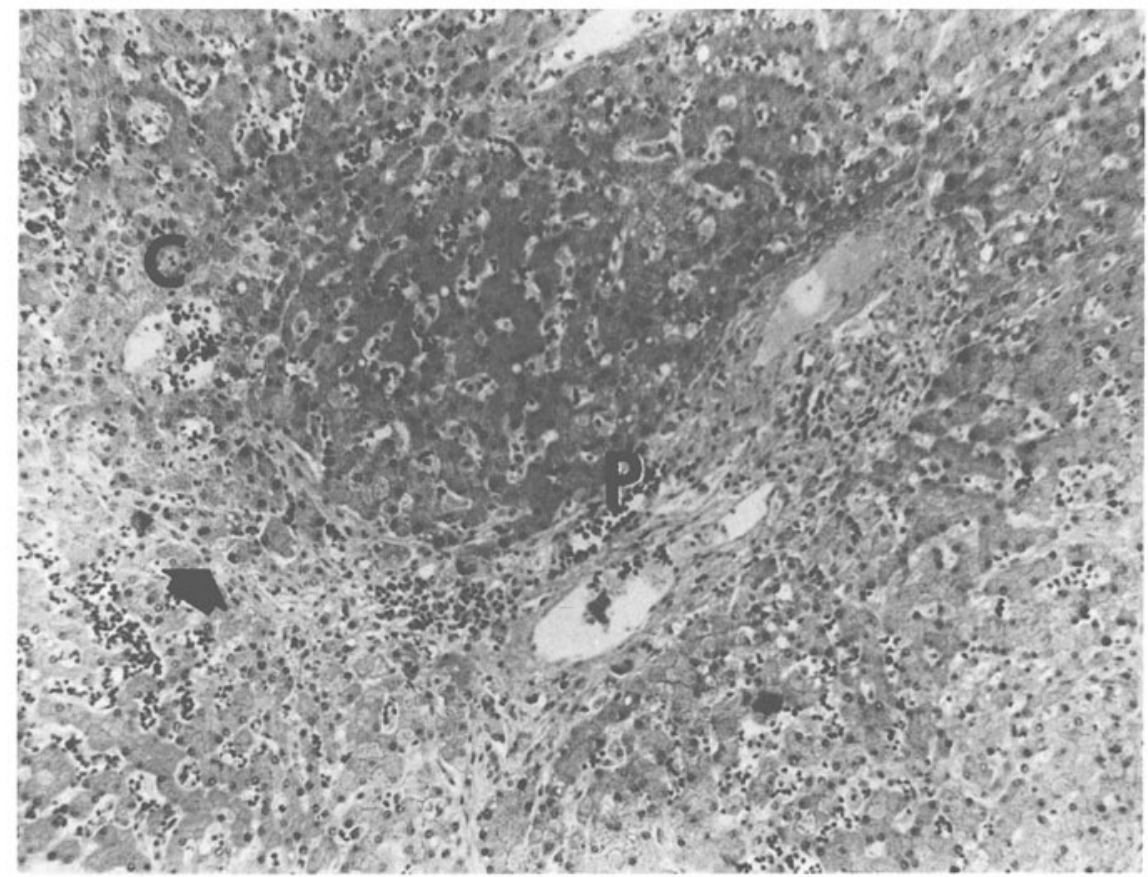

Fig 8. A central vein (C) and a portal tract (P) are connected by a delicate fibrous septa (arrow) (Masson trichrome, $\times 90$ ).

local ischemia. The septate scars and irregular nodules obstruct the portal venous system, thus leading to portal hypertension (8).

\section{The Nomenclature of Cirrhosis}

Classification systems of cirrhosis are numerous, and names for various types overlap, creating a confusion of nomenclature. Gall's classification attempted to relate etiology to

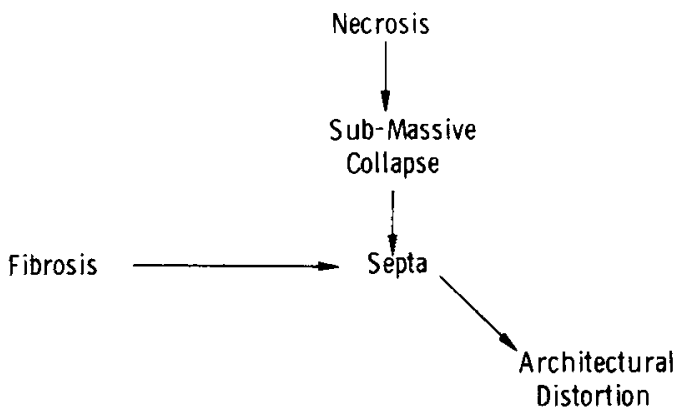

Fig 9. Collapse and septation. morphology and used such names as nutritional, postnecrotic and posthepatitic to define specific forms of cirrhosis (9). Rubin and Popper simplified this considerably by treating all cirrhosis, regardless of cause, as belonging to one of two morphologic types, based on the size of the regenerative nodules which was thought to reflect the intensity of the insult (2). Cirrhosis, characterized by nodules which were fragments of preexisting lobules, was thought to be due to a continuous injury and was classified as monolobular, while that with nodules composed of more than one preexisting lobule was called mutilobular and was thought to be due to a discontinuous injury $(2,10)$. Scheuer's system of micronodular and macronodular cirrhosis (11) was somewhat similar to that of Rubin and Popper.

The value of any classification resides in its recognition of etiology, with specification of morphology as of secondary importance (12). It 


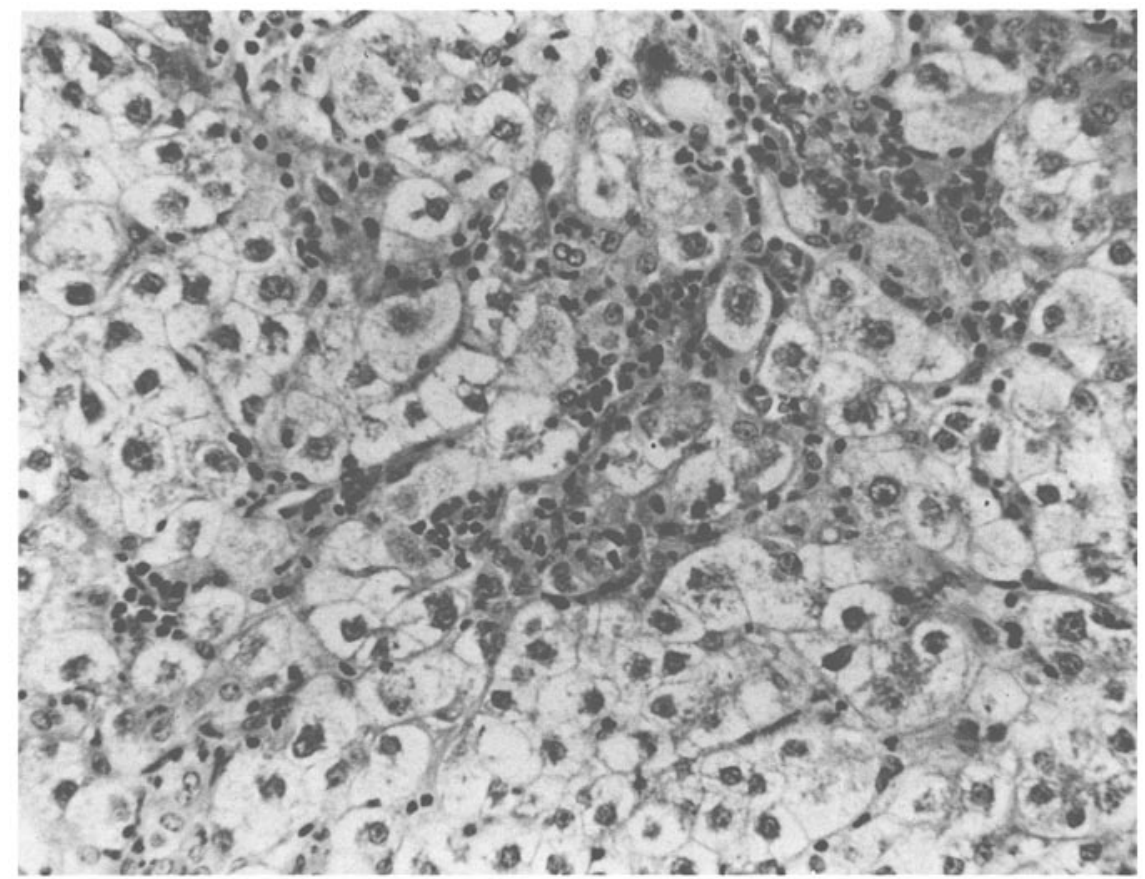

Fig 10. Viral hepatitis: scattered among the distorted hepatic cells are small clusters of inflammatory cells, evidence of very focal necrosis and collapse $(H \& E, \times 225)$.

is important to recognize that a particular morphologic pattern of cirrhosis is not necessarily a static condition, but patterns may change, probably under the influence of nutritional factors, a persisting original insult or the change may be instigated by new or secondary insults $(2,11)$. For instance, the chronic alcoholic typically developed a cirrhosis with fine septa, diffusely dividing the liver into small sublobular fragments. We now see more coarsely nodular cirrhosis, with thicker septa in alcoholics, perhaps related to better nutrition.

An ideal system would define cirrhosis according to cause, perhaps using terms as "cirrhosis of chronic alcoholism," or "cirrhosis of viral hepatitis" or "cirrhosis of biliary tract obstruction." As it stands now, any known cause of cirrhosis can produce just about any morphologic pattern (13), and for many cases of cirrhosis, the causes are unknown. Therefore, for the present, and until we can pinpoint etiology better, no specific classification system is recommended; that which allows the clearest communication between clinicians and pathologists is the best. When a clinician suspects his alcoholic patient has portal or Laennec's or nutritional cirrhosis, but is told by the pathologist that the patient's liver is actually an example of postnecrotic cirrhosis-whether or not it is loaded with fat and Mallory bodies-it is obvious that classifications utilizing the terms portal or nutritional or postnecrotic are only creating barriers to communication. What is important is the recognition that this cirrhosis is of a type which can occur in an alcoholic, whatever name it is given.

Finally, one more aspect of communication needs clarification-the concept of cirrhosis as a point in time. To place all progressive hepatic disease in perspective, we must consider a spectrum of liver diseases, at one end of which there is a scarred, nodular, distorted entity known as 


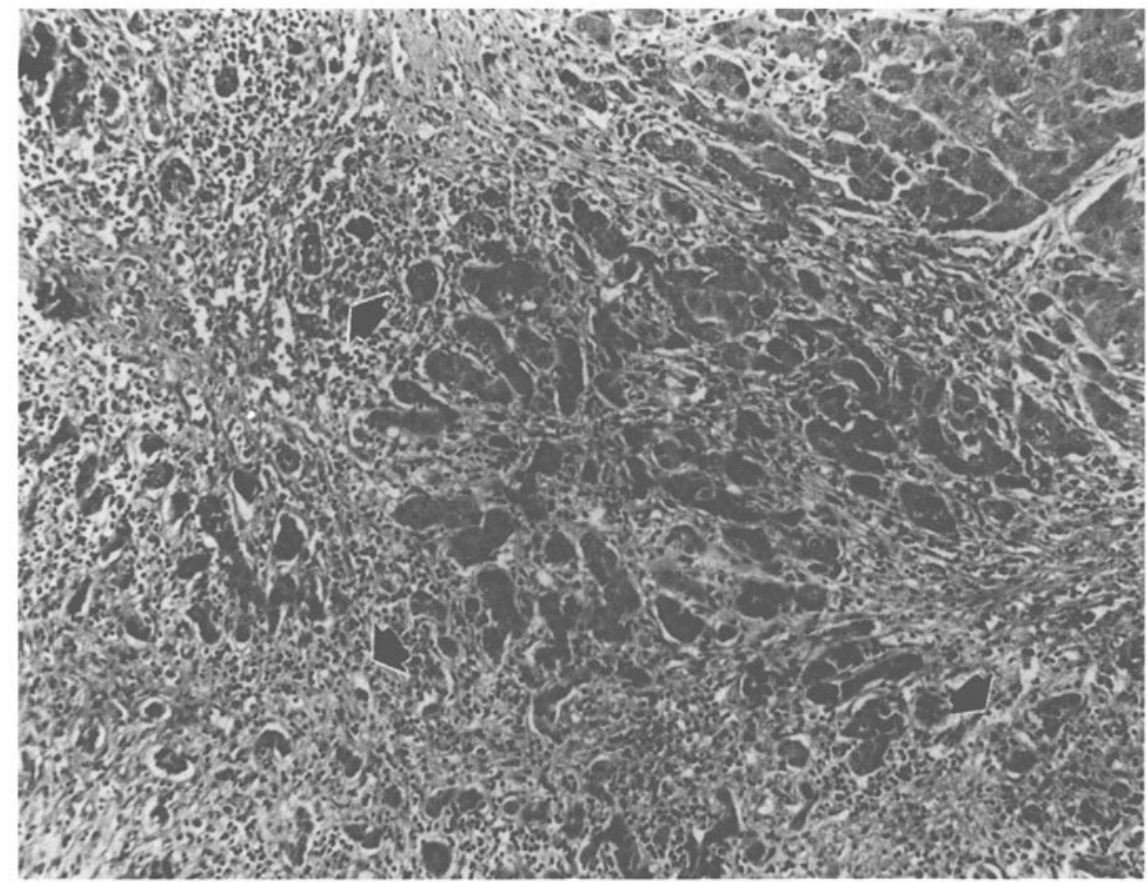

Fig 11. Submassive hepatic necrosis: the arrows point to several large foci of collapse characterized by finely fibrillar collagen. small inflammatory cells and many bile ducts seen in cross section $(H \& E, \times 90)$.

"cirrhosis," and at the other end, is a normal liver, insulted for the first time by one or more of a variety of infectious, toxic, metabolic or mechanical agents. Between them there are an infinite number of intermediate stages. Some of these transitional conditions already have names such as: subacute hepatic necrosis (7), chronic aggressive hepatitis (5), alcoholic hepatitis (14), steatonecrosis of Mallory body

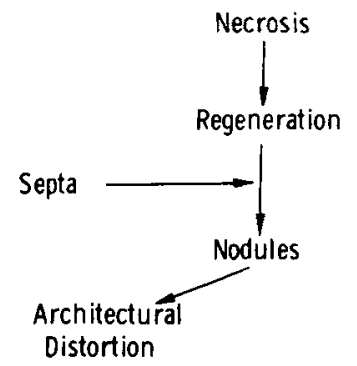

Fig 12. Regeneration and nodule formation. type (15), chronic nonsuppurative cholangitis (16) and even something called early cirrhosis (15). Somewhere along this spectrum, the clinical features of cirrhosis develop, although full distortion of morphologic cirrhosis may not be present at this point, yet, ultimately, it too is likely to appear. There is no magic moment, either clinical or morphologic, when cirrhosis suddenly exists. The transitional stages are gradual. Whether or not a given patient has actually reached the point of cirrhosis is generally an almost arbitrary judgment on the part of the clinician and/or pathologist.

\section{REFERENCES}

1. Schaefer JW, Schiff L, Gall EA, et al. Progression of acute hepatitis to post-necrotic cirrhosis. Am J Med 42:348-358, 1967

2. Rubin E, Popper $\mathbf{H}$ : The evolution of human 


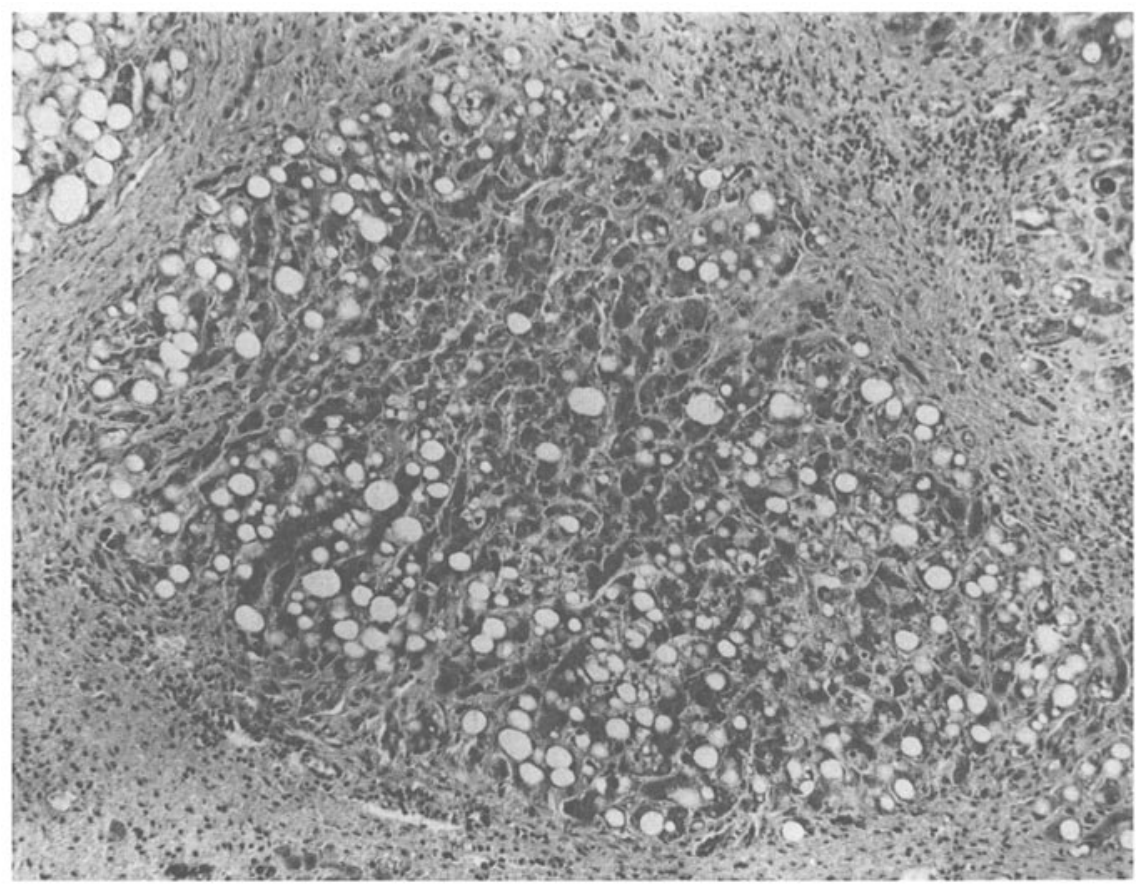

Fig 13. A nodule of regenerating hepatocytes surrounded by scar. The clear spaces are lipid vacuoles in the liver cells (Masson trichrome, $\times 90$ ).

cirrhosis deduced from observations in experimental animals. Medicine 46:163-183, 1967

3. Popper H: The problem of hepatitis. Am J Gastroenterol 55:335-346, 1971

4. Popper H, Udenfriend S: Hepatic fibrosis: correlation of biochemical and morphologic investigations. Am J Med 49:707-721, 1970

5. Alpert LI, Vetancourt R: Chronic hepatitis. A clinicopathologic study of 75 cases. Arch Path 88:593-601, 1969

6. Edmondson HA, Peters RL, Frankel HH, et al: The early stage of liver injury in the alcoholic Medicine 46:119-129, 1967

7. Boyer JL, Klatskin G: Patterns of necrosis in acute viral hepatitis: Prognostic valve of bridging (subacute hepatic necrosis). N Engl J Med 283:1063-1070, 1970

8. Baggenstoss AH: Post-necrotic cirrhosis. Morphology, etiology, and pathogenesis, Progress in Liver Disease, New York, Grune and Stratton, 1961, pp 14-38
9. Gall EA: Posthepatitic, postnecrotic, and nutritional cirrhosis: A pathologic analysis. Am J Pathol 36:241-258, 1960

10. Hellstrom HR, Fisher ER: Cirrhosis, A complex clinicopathologic spectrum (Abst) Am J Clin Pathol 52:755, 1969

11. Scheuer DJ: Liver Biopsy Interpretation. London, Bailliere, Tindall and Cassell, 1970, pp 55-69

12. Patek AJ, Jr: Diseases of the Liver. Edited by L Schiff Third edition. Philadelphia, JB Lippincott Co, 1969, pp 689-738

13. Popper $H$, Schaffner $F$ : Hepatic cirrhosis: A problem in communication. Isr J Med Sci 4:1-7, 1968

14. Porta EA, Bergman BJ, Stein AA: Acute alcoholic hepatitis. Am J Pathol 46:657-689, 1965

15. Harinasuta $U$, Chomet $B$, Ishak $K$, et al: Steatonecrosis: Mallory body type. Medicine 46:141-162, 1967

16. Foulk WT, Baggenstoss AH:12 pp 739-769 